\title{
A STUDY OF CLINICAL PRESENTATIONS IN DIABETIC FOOT AND EVALUATION OF THE OUTCOME OF VARIOUS TREATMENT STRATEGIES EMPLOYED AT OUR INSTITUTE
}

\author{
Ajit Hemant Nehete', Puja Bhaurao Dandekar ${ }^{2}$ \\ 1 Lecturer, Department of General Surgery, Dr. PDMMC, Amravati, Maharashtra, India. \\ ${ }^{2}$ Senior Resident, Department of General Surgery, Dr. PDMMC, Amravati, Maharashtra, India.
}

\begin{tabular}{l}
\hline ABSTRACT \\
BACKGROUND \\
Diabetic foot is one of the most feared complications of diabetes, with prolonged duration of hospitalization, high expenses with an \\
eventuality of amputation of limb. There are approximately 50,000 to 60,000 amputations performed in diabetic patients each \\
year. The treatment of diabetic wounds involves local and systemic measures. The aim of the study is to analyse various modes of \\
clinical presentations of diabetic foot and to evaluate the outcome of various treatment strategies employed at our institute.
\end{tabular}

\section{METHODS}

A total of 50 clinically diagnosed cases of diabetic foot were studied over a period of 18 months in tertiary care hospital, central India. Institutional ethical committee approval was obtained to carry out this study. Informed consent was obtained from all the patients included in the study. In the present study, we intended to study the signs, symptoms, and complications of the disease, and also management of diabetic foot. Sample size was taken for convenience.

\section{RESULTS}

Male preponderance was seen in our study (84\%). Maximum number of patients were seen in the age group of 51 to 60 years (44\%). Most common initiating factor was trauma of some kind in $64 \%$ of the cases. Most common mode of presentation was ulcers (56\%) and cellulitis (26\%). In some patients, only control of diabetes with insulin along with appropriate antibiotics was effective. Thorough surgical debridement with daily dressings was helpful. Other modes of management included skin grafting, disarticulation, amputation.

\section{CONCLUSIONS}

It is possible to reduce incidence and complications of diabetic foot through early diagnosis, appropriate management, and prevention programs.

HOW TO CITE THIS ARTICLE: Nehete AH, Dandekar PB. A study of clinical presentations in diabetic foot and evaluation of the outcome of various treatment strategies employed at our institute. J. Evolution Med. Dent. Sci. 2019;8(19):1533-1536, DOI: $10.14260 /$ jemds/2019/340

\section{BACKGROUND}

Diabetes mellitus (DM) is one of the most important and common metabolic disorders affecting about $2-5 \%$ of the population in Europe and about $20 \%$ of the population in various other parts of the world,[1] diabetes mellitus (DM) is one of the most important and common metabolic disorders affecting about $2-5 \%$ of the population in Europe and about $20 \%$ of the population in various other parts of the world.[1] Diabetes mellitus (DM) is one of the most important and common metabolic disorders affecting about $2-5 \%$ of the population in Europe and about $20 \%$ of the population in various other parts of the world.[1] Diabetes mellitus (DM) is one of the most important and common metabolic disorders affecting about $2-5 \%$ of the population in Europe and about $20 \%$ of the population in various other parts of the world.[1]

Diabetes Mellitus (DM) is one of the most widespread non communicable diseases across the world.

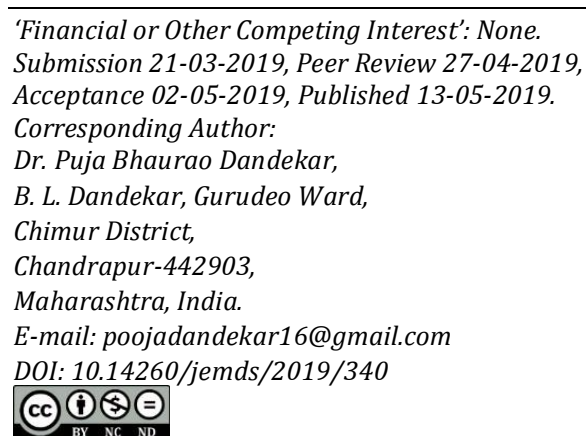

The incidence of diabetes mellitus is increasing worldwide; by 2030, it will grow up to 366 million. This estimation occurred because of longer life expectancy and changing habits of diet.[1]

Diabetes mellitus (DM) is one of the most important and common metabolic disorders affecting about $2-5 \%$ of the population in Europe and about $20 \%$ of the population in various other parts of the world.[1]

Even though there are many complications affecting the person with diabetes, none are more devastating than those complications involving the foot.[2] Two types of complications are encountered usually with DM: microvascular and macrovascular. Diabetic neuropathy is one of the most commonly-occurring microvascular complications, of which the most common type is distal symmetrical neuropathy or polyneuropathy. It is estimated that $15 \%$ of patients with diabetes will suffer from diabetic foot ulcer during their lifetime. [3]

The relationship between diabetic neuropathy, the insensitive foot, and foot ulceration was recognized by Pryce, a British surgeon, over a century ago. He stated that, "It was abundantly evident that the actual cause of the perforating ulcer was peripheral nerve degeneration and that diabetes itself played an active part in the causation of the perforating ulcer.[4]

Foot ulcers significantly contribute to morbidity and mortality of patients with diabetes mellitus. The diabetic patients with foot ulcers require long-term hospitalization and carry the risk of limb amputation.[5] Studies show that 
severity of diabetic foot ulcer is the strongest significant risk factor of amputation for diabetes patients. In developed countries, one in every six people with diabetes will have an ulcer during their lifetime. The risk is even higher in developing countries.[6]

In total, the most common pathway to develop foot problems in patients with diabetes is peripheral sensory motor and autonomic neuropathy that leads to high foot pressure, foot deformities, and gait instability, which increases the risks of developing ulcers.[7] Today, numerous investigations have shown that elevated plantar pressures are associated with foot ulceration. ${ }^{[8]}$ Additionally, it has been demonstrated that foot deformities and gait instability increases plantar pressure, which can result in foot ulceration.[9]

Multiple risk factors associated with the development of DF.[10] These risk factors are as follows: gender (male), duration of diabetes longer than 10 years, advanced age of patients, high Body Mass Index, and other co-morbidities such as retinopathy, diabetic peripheral neuropathy, peripheral vascular disease, glycated haemoglobin level (HbA1C), foot deformity, high plantar pressure, infections, and inappropriate foot self-care habits.[11] The primary management goals for DF are to obtain wound closure as expeditiously as possible.[12] As diabetes is a multi-organ systemic disease, all co-morbidities that affect wound healing must be managed by a multidisciplinary team for optimal outcomes.

In India, it is estimated that approximately 40, 000 legs are being amputated every year, of which $75 \%$ are neuropathic with secondary infection, which is potentially preventable. Infection of the foot is the frequent complication of diabetes mellitus and affects $12 \%$ of patients with diabetes mellitus. By taking certain measures like regular inspection of foot daily for redness, swelling and other signs of injury, wearing appropriate shoes, cessation of smoking etc one can prevent the diabetic foot problem and its complications.

Hence this study has been undertaken to evaluate the various modes of clinical presentation of diabetic foot ulcer and to evaluate the outcome of various treatment strategies.

\section{METHODS}

This is an observational study. A total of 50 clinically diagnosed cases of diabetic foot were studied over a period of 18 months in tertiary care hospital, central India. Institutional ethical committee approval was obtained to carry out this study. Informed consent was obtained from all the patients included in the study. In the present study we intended to study the signs, symptoms and complications of the disease and also management of diabetic foot. This study was conducted in tertiary care hospital, central India, which included total 50 cases of diabetic foot who presented in surgery OPD or emergency department and got admitted. Sample size was taken for convenience.

\section{Inclusion Criteria}

1. Any known diabetic patient with chronic foot and leg ulcers.

2. Any known diabetic patient with Cellulites, osteomyelitis, blisters, gangrene of lower limb.
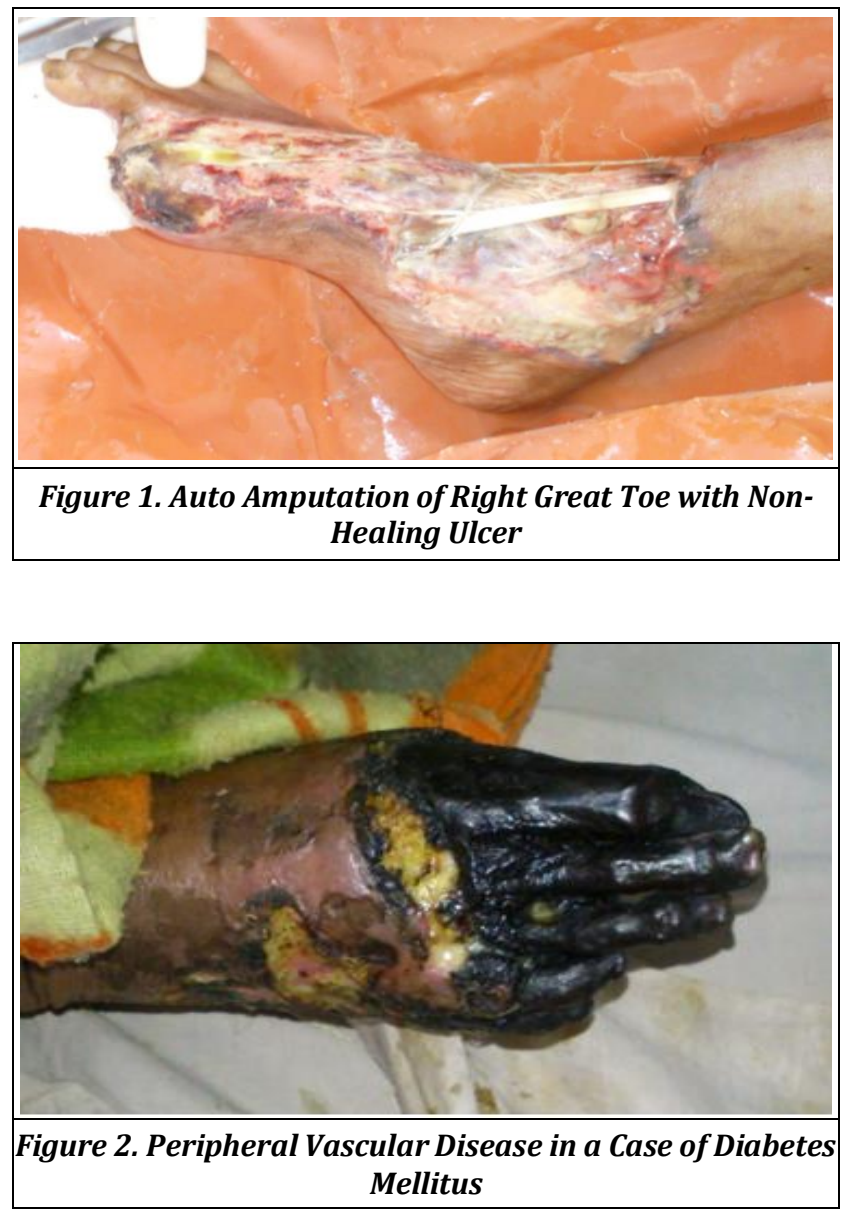

3. Patients with all the above clinical presentations diagnosed as diabetic, after admission (Random blood sugar-more than $160 \mathrm{mg} / \mathrm{dl}$, Fasting blood sugar-more than $100 \mathrm{mg} / \mathrm{dl}, 2 \mathrm{hrs}$. Postprandial-more than 140 $\mathrm{mg} / \mathrm{dl}) \cdot[13]$

\section{Exclusion Criteria}

Patient aged below 18 years.

\section{Statistical Analysis}

The data were reported as mean \pm SD and frequency. The data collected was entered into Microsoft excel spread sheet and analysed using IBM SPSS Statistics, Version 16.

\section{RESULTS}

Age

Out of 50 patients studied, most of the diabetic patients were in the age group of 51-60 (44\%).The youngest patient was of 35 years and the oldest was of 80 years.

\section{Sex Distribution}

In present study out of 50 patient 42 were male and 8 were females. It shows male predominance. $42(84 \%)$ were male patient and $8(16 \%)$ cases were female.

\section{Clinical Presentation}

In present study of the 50 cases, $56 \%$ presented with ulcer, $26 \%$ cellulitis, $10 \%$ presented with ulcer with gangrene and $8 \%$ with ulcer with cellulitis. 


\section{History of Trauma}

In present study history of trauma (thorn prick, shoe bite, nail prick, wood piece prick etc. as a precipitating factor was present in 32 patients making a total of $64 \%$.

\section{Site of Lesion}

Most common site of lesion was dorsum of foot accounting for $38 \%$ followed by toes accounting for $28 \%$.

\section{Duration of Diabetes Mellitus}

In present study, 17 patients were detected as diabetes mellitus on admission and rest 33 patients were known case of diabetes. In 18 patients, duration of diabetes mellitus was between 1-5 years.

\section{Incidence of Osteomyelitis}

Osteomyelitis was seen in $\mathrm{x}$-rays of 6 patients.

\section{Incidence of Peripheral Neuropathy}

Out of 50 patients, 22 patients with diabetic foot had peripheral neuropathy.

\section{Incidence of Different Causative Organisms}

The most common organism grown on culture of pus was Staphylococcus aureus in 28 (56\%) patients followed by Pseudomonas in 6 patients (12\%), Proteus in 5 patients, E coli in 3 patients, Klebsiella in 4 patients. In 4 patients (8\%) there was no growth.

\section{Glycosylated Hb (HbA1c) Level}

Out 50 patients, 33 patients accounting for $66 \%$ showed glycosylated $\mathrm{Hb}$ level less than $7 \mathrm{mg} \%$ and had better glucose control with shorter duration of hospital stay compared to 17 patients in whom glycosylated $\mathrm{Hb}$ were more than $7 \mathrm{mg} \%$ and had poor glucose control.

\section{Duration of Hospital Stay}

The average duration of hospital stay was 22 days with minimum days of stay of 5 days and the maximum days of stay being 90 days.

\section{Surgical Treatment}

In present study, debridement was done in 32 patients, skin grafting after thorough debridement was done in 6 patients, and major amputation in 5 patients. Only dressings were done in 4 patients.

\section{DISCUSSION}

In the present study we have studied total 50 cases of diabetic foot. Most common age group who presented with diabetic foot in my study was between $51-60$ years with an average of 56.4 years.

In Study of Reiber et al the highest percentage was in persons aged between 45 to 64 years.[17] In present study, 42 were male and 8 were female, out of 50 , comprising of $84 \%$ males and $16 \%$ females.

In Manchester series study, $67.4 \%$ were male and $32.6 \%$ were female.[18] Male preponderance may be due to males are more exposed to injuries during their occupational and recreational activities that put more stress on the feet In my study majority of patients presented with Ulcer (56\%), 26\% with cellulitis, $10 \%$ with ulcer and gangrene and $8 \%$ with cellulitis with ulcer.

Description of the ulcer characteristics on presentation is essential for the mapping of the ulcer's progress during treatment.[19,20]

In the present study $64 \%$ of patients were presented with history of trauma. This was comparable to Reiber et al series in which $77 \%$ of patients had history of trauma.[18]

Out of 50 patients studied, most common site of lesion was dorsum of foot (38\%) followed by toes $(28 \%)$ and sole of foot $(22 \%)$ of all cases.

As per Apelquist Study, toes were affected in $51 \%$, sole was affected in $28 \%$ and dorsum in $14 \%$. [21]

Out of 50 patients, 18 patients presented between 1-5 years and mean age is 2.5 years. 17 patients were detected diabetes mellitus on admission.

This shows that foot complications occurred early in our study most probably due to lack of strict glucose control.

In the present study $12 \%$ of the patients had osteomyelitis. In the x-ray. Demineralization, periosteal reaction and bony destruction are classic radiographic triad of osteomyelitis. It is comparable to Manchester series in which $20 \%$ had osteomyelitis and JOS university study,[18,22] in which $14 \%$ had osteomyelitis.

The incidence of peripheral neuropathy in present study is $44 \%$ that is out of 50 patient, 22 patients were having peripheral neuropathy. In Walter DP et al series, the incidence of peripheral neuropathy was $39.4 \%$.

In the present study, pus discharge and swab from ulcer were sent for culture and antibiotic sensitivity testing.

Most common organism isolated was staphylococcus aureus in 28 patients out of 50, comprising of $56 \%$.

Other organisms isolated were pseudomonas (12\%), proteus (10\%), Klebsiella (8\%) and E. coli (6\%). In $8 \%$ patients, there was no growth of any bacteria.

Infection is the 3rd most common factor responsible in the pathogenesis of diabetic foot lesion. In the present study only 33 patients had $\mathrm{HbA1c}$ level less than $7 \mathrm{mg} \%$ and they had shorter duration of hospital stay compared to the patients with HbA1c more than $7 \mathrm{mg} \%$. In the present study, average duration of hospital stay was 22 days. The duration of hospital stay was related to control of blood sugar level and type, extent, severity of disease.

In present study, out of 50 patients, 32 patients underwent thorough debridement. STSG was done in 6 patients with good uptake of graft. STSG reduced the healing time in these patients and decreased their hospital stay.

Conservative treatment along with daily dressing was done in 4 patients having less infected ulcer. Disarticulation of toes in 3 patients was done. Amputation was done in 5 patients with complicated foot infection.

\section{CONCLUSIONS}

Patients with diabetes mellitus are often encountered with complications such as ulceration, infection, gangrene, cellulitis and lower extremity amputations. These complications frequently result in extensive morbidity, repeated hospitalizations and mortality. They take a tremendous toll on the patient's physical and mental wellbeing as well as impose a substantial economic burden, often removing the patient from the workforce and placing a financial drain on the health care system. Not all diabetic foot 
complications can be prevented, but it is possible to dramatically reduce their incidence through appropriate management and prevention programs. Such prevention programs can reduce the incidence of foot ulceration through modification of self-care practices, appropriate evaluation of risk factors, and formulation of treatment protocols aimed at early intervention, limb preservation, and prevention of new lesions.

\section{REFERENCES}

[1] Saad N, Elhadedy K, Ramadan N, et al. The prevalence and risk categorization of diabetic foot complications in cohort group in, Beni Suif, Egypt. Life Science Journal 2013;3:10.

[2] Jain AKC. A new classification of diabetic foot complications: a simple and effective teaching tool. The Journal of Diabetic Foot Complications 2012;4(1):1-5.

[3] Leone S, Pascale R, Vitale M, et al. Epidemiology of diabetic foot. Infez Med 2012;20 Suppl 1:8-13.

[4] Pryce TD. A case of perforating ulcers of both feet associated with diabetes and ataxic symptoms. Lancet 1887;130(3331):11-2.

[5] Nyamu PN, Otieno CF, Amayo E0, et al. Risk factors and prevalence of diabetic foot ulcers at Kenyatta National Hospital, Nairobi. East African Medical Journal 2003;80(1):36-43.
[6] Viswanathan V, Kumpatla S. Pattern and causes of amputation in diabetic patients - a multicentric study from India. Journal of the Association of Physicians of India 2011;59:148-51.

[7] Formosa C, Gatt A, Chockalingam N. Diabetic foot complications in Malta: prevalence of risk factors. Foot (Edinb) 2012;22(4):294-7.

[8] Ledoux WR, Shofer JB, Cowley MS, et al. Diabetic foot ulcer incidence in relation to plantar pressure magnitude and measurement location. J Diabetes Complications 2013;27(6):621-6.

[9] Bacarin TA, Sacco IC, Hennig EM. Plantar pressure distribution patterns during gait in diabetic neuropathy patients with a history of foot ulcers. Clinics (Sao Paulo) 2009;64(2):113-20.

[10] Frykberg RG, Zgonis T, Armstrong DG, et al. Diabetic foot disorders. A clinical practice guideline (2006 revision). J Foot Ankle Surg 2006;45(Suppl 5):S1-S66.

[11] McEwen LN, Ylitalo KR, Herman WH, et al. Prevalence and risk factors for diabetes-related foot complications in Translating Research Into Action for Diabetes (TRIAD). J Diabetes Complications 2013;27(6):588-92.

[12] Ramachandran A. Urban India: a breeding ground for diabetes. Diabetes Voice 2002;47(1):18-20.

[13] Longo D, Kasper D, Jameson LJ, et al. Harrison's Principles of Internal Medicine. 17th edn. McGraw-Hill Professional 2008;2:2275. 